\title{
Human adipose-derived stromal/stem cells demonstrate short-lived persistence after implantation in both an immunocompetent and an immunocompromised murine model
}

Hitesh Agrawal ${ }^{1,5^{*}}$, Hulan Shang ${ }^{2,5}$, Anna Parker Sattah ${ }^{3,5}$, Ning Yang ${ }^{2,5}$, Shayn M Peirce ${ }^{4}$ and Adam J Katz ${ }^{2,5}$

\begin{abstract}
Introduction: Mesenchymal cells are emerging as a promising cell platform for regenerative therapies. However, the fate of cells after transplantation in many different disease settings and tissue beds remains unclear.

Methods: In this study, human adipose-derived stromal/stem (ASCs) cells were fluorescently labeled with a membrane dye and injected into both immunocompetent and immunocompromised mouse strains. Cells were injected either as single cell suspensions, or as self-assembling spheroids. In parallel, cells were purposefully devitalized prior to injection and then implanted in the opposite side in a randomized fashion. These 'control' groups were included to determine whether the fluorescent membrane dye would remain localized at the injection site despite the use of nonviable cells. Cell implants and the surrounding tissues were harvested on days 3, 10 and 21 after in vivo delivery and evaluated in a blinded manner. Injection sites were analyzed by fluorescent microscopy, and human cell numbers were quantified using PCR detection of a human-specific endogenous retrovirus (ERV-3). Host response was evaluated by immunofluorescent staining of macrophages.
\end{abstract}

Results: ERV-3 quantification showed that 95\% of the human cells that were viable when they were injected were undetectable at the three-week time-point. Although fluorescent signal persisted for the entire study period, further analysis revealed that much of this signal was located within host macrophages.

Conclusions: These results suggest that human ASCs survive for less than three weeks after injection into even immunocompromised mice, and call into question the notion that human ASCs are immuno-privileged and capable of surviving for extended periods in xenogeneic and/or allogeneic models.

\section{Introduction}

As the promise of cell-based therapies begins to transition to the clinic, a clear understanding of the survival, localization and identity of administered cells over time remains elusive but of great interest. A major limitation relates to various technical challenges associated with the reliable identification and tracking of cells in vivo. Even in the context of preclinical animal models, the sensitive and specific identification of human cells after

\footnotetext{
* Correspondence: hiteshdos@hotmail.com

1 Department of Pediatric Cardiology, Baylor College of Medicine, Texas

Children's Hospital, 6621 Fannin Street, Houston, TX 77030, USA

${ }^{5}$ Department of Plastic Surgery, University of Virginia, 1215 Lee Street,

Charlottesville, VA 22903, USA

Full list of author information is available at the end of the article
}

implantation presents certain challenges. Although a number of methods have been described, most - if not all - should be re-evaluated in the context of recent findings related to cell-derived vesicles, or microparticles $[1,2]$. These elements encompass a heterogeneous spectrum of membrane-bound structures ranging in size from $20 \mathrm{~nm}$ to $1 \mu \mathrm{m}$, released by an ever-expanding list of cell types, and containing DNA, RNA, and cytosolic and/or membrane-associated proteins [2]. The notion that such microvesicles may play a role in the false positive contamination of host cells by labeled donor cells has been reported recently in the literature [3].

A variety of literature suggests that human adiposederived stromal/stem cells (ASCs) are immunotolerant/ immunoprivileged and can survive for prolonged periods 
in immunocompromised and even immunocompetent animals [4-7]. In this study we investigated the survival of human ASCs in both immunocompetent and immunocompromised murine models, delivered either as singlecell suspensions or as self-assembled three-dimensional spheroids. The extent of human cell engraftment was quantified over time using PCR detection of an endogenous retrovirus (ERV-3) present in all human cells, but not present in rodent cells. Since the ERV-3 genetic sequence is present as only a single copy per human cell, its detection is directly proportional to the number of human cells present. Using this sensitive and specific method of human cell detection, we demonstrate that nearly $90 \%$ of human ASCs that were viable upon injection are undetectable by day 10 after administration into nude mice. Human cell clearance is even faster in immunocompetent animals.

\section{Materials and methods}

\section{Isolation and culture expansion of human} adipose-derived stromal/stem cells

Human adipose tissue samples were obtained from elective surgical procedures under Institutional Review Board approval at the University of Virginia. We obtained all necessary consent from any patients involved in the study. ASCs were isolated as described previously [8,9]. Briefly, samples were washed, enzymatically dissociated with Liberase Blendzyme (Roche Applied Science, Indianapolis, USA), and filtered to remove debris [9]. After centrifugation, pelleted cells were recovered and washed. Contaminating erythrocytes were removed by osmotic buffer, and the cells were plated onto tissue culture plastic and culture expanded in adherent monolayer culture in xenogeneicfree growth medium with 1\% human serum (LM1\%) [10]. After three passages, culture-expanded ASCs were membrane dye (DiI) labeled according to the manufacturer's protocol (Molecular Probes, Inc., Eugene, OR, USA). Some labeled cells were formed into self-assembling spheroids in hanging drop [11,12]. Mouse embryonic fibroblast cells (3 T6) were obtained from University of Virginia cell culture core facility and were cultured in Dulbecco's modified Eagle's medium/F12 high glucose with $10 \%$ fetal bovine serum.

\section{Dil labeling of adipose-derived stromal/stem cells}

ASCs were suspended at a density of $1 \times 10^{6} / \mathrm{ml}$ in culture medium. Five microliters of DiI cell labeling solution were added to the cell suspension, followed by gentle mixing with a pipette. The mixture was incubated at $37^{\circ} \mathrm{C}$ for 20 minutes. Next, the labeled suspension tubes were centrifuged at $1,500 \mathrm{rpm} / 200 \times g$ force for 5 minutes at $37^{\circ} \mathrm{C}$. This was followed by removal of supernatant and resuspension of the cells in Dulbecco's modified Eagle's medium/F12 high glucose with 10\% fetal bovine serum at $37^{\circ} \mathrm{C}$. This washing procedure was repeated two more times.

Adipose-derived stromal/stem cell implantation into mice Procedures were performed with approval of the University of Virginia Animal Care and Use Committee. Two strains of mice were used. Thirty-six immunocompetent wildtype (C57BL/6NCr) mice and 36 immunocompromised (Athymic $\mathrm{NCr}-\mathrm{nu} / \mathrm{nu}$ ) mice were anaesthetized using ketamine and randomly treated with 300,000 cells either in suspension or preaggregated into spheroids (10 spheroids each comprised of 30,000 cells), followed by appropriate postoperative pain control. Cells delivered as suspensions were injected subcutaneously and into the inguinal region, while cells formulated as three-dimensional spheroids were delivered through an incision into the inguinal fat pad of mice. Implants composed of nonviable cells/spheroids served as parallel controls, implanted in the contralateral side in a randomized, blinded fashion. Nonviable cell implants were generated by overnight incubation at $-80^{\circ} \mathrm{C}$, thawing at room temperature and confirmed as nonviable with trypan blue dye exclusion and Cell Proliferation Reagent WST-1 (Roche Applied Science).

\section{Harvesting and processing of tissues}

Three sets of mice (each set comprising 12 immunocompetent mice and 12 immunocompromised mice) were harvested on days 3,10 and 21 after implantation. Through random sampling, one-half of the mice from each harvest time point were assigned to be used for histology and one-half of the mice for human cell quantification by PCR detection of ERV-3. The histology specimens were fixed in 10\% neutral buffered formalin and were embedded in paraffin while the PCR samples were frozen at $-80^{\circ} \mathrm{C}$.

\section{Quantification of human adipose-derived stromal/stem cells}

Real-time PCR detection of the human/primate-specific ERV-3 was used to evaluate and quantify the presence of human ASCs. Of note, the ERV-3 gene is known to reside at a single locus (on human chromosome 7), enabling a direct correlation between ERV-3 levels and human cell numbers [13]. The primers for the human specific ERV-3 gene were designed as described previously $[14,15]$ : forward, 5-ATG GGA AGC AAG GGA ACT AAT G; reverse, 5-CCC AGC GAG CAA TAC AGA ATT T (Integrated DNA Technologies, Coralville, Iowa, USA). Preserved samples from injection sites were frozen with liquid nitrogen and ground to powder using a mortar and pestle. DNA extraction was performed with DNAzol (Molecular Research Centre, Cincinnati, Ohio, USA) according to the manufacturer's protocol. DNA extract from cultured ASCs served as a positive control (that is, ASCs 
$100 \%)$ and DNA extract from an untreated mouse was used as a negative control (that is, ASCs 0\%).

Standards were prepared by combining cultured human ASCs and mouse embryonic fibroblast cells (3 T6) in known ratios (ASCs 4.76\%, 0.498\%, 0.05\%, and 0.005\%). Accordingly, standards were generated from the mixture of defined numbers $\left(5 \times 10^{4}, 5 \times 10^{3}, 5 \times 10^{2}\right.$ and $\left.5 \times 10\right)$ of human ASCs with $10^{6}$ mouse embryonic fibroblast cells. Genomic DNA was extracted from these preparations according to experimental protocol. Real-time quantitative PCR with 96-well optical plates was performed and analyzed using an icycler iQ (BioRad, Hercules, CA, USA). Each reaction was performed using $4.5 \mu \mathrm{l}$ DNA specimen added to $8 \mu \mathrm{l}$ PCR reagent mixture comprised of SYBR green and forward and reverse primers. Extracted DNA was assessed for quality and quantity using GeneQuant Pro (Amersham Biosciences, Piscataway, NJ, USA) and each sample was run at 1:10 and 1:50 dilutions in duplicate. The PCR conditions used were: first step, $95^{\circ} \mathrm{C}$ for 15 minutes; and second step, 45 cycles each with $30 \mathrm{sec}-$ onds at $95^{\circ} \mathrm{C}$ (denaturation), 30 seconds at $60^{\circ} \mathrm{C}$ (annealing) and 30 seconds at $72^{\circ} \mathrm{C}$ (extension). The threshold cycle $\left(\mathrm{C}_{\mathrm{T}}\right)$ was defined as the first cycle number in a PCR amplification above baseline and during the exponential increase period, with 40 as the maximum allowable value. Appropriate amplification was determined by melt curve analysis, with an ERV-3 melting temperature of $87.5^{\circ} \mathrm{C}$.

For generation of a standard curve, six defined mixtures/ratios of human ASCs and mouse embryonic fibroblast cells (3 T6) were analyzed. The amplification curves and $C_{T}$ values obtained are presented in Table 1. Log[human ASCs] linearly correlated with $\mathrm{C}_{\mathrm{T}}$ values, with a correlation coefficient of 0.97 (Figure 1). From these results, an equation depicting the relationship between $\mathrm{C}_{\mathrm{T}}$ values and human ASCs was obtained as follows:

Human ASC number $=10^{-\left(\mathrm{C}_{\mathrm{T}}^{-39.5}\right) / 3.03}$

Table 1 Real-time PCR results

\begin{tabular}{lcccc}
\hline Mouse 3 T6 cells & Human ASCs & Human ASC \% & $\boldsymbol{C}_{\mathbf{T}}(\boldsymbol{n}=\mathbf{6})$ & STD \\
\hline $1,000,000$ & 50,000 & 4.760 & 24.6 & 0.3884 \\
$1,000,000$ & 5,000 & 0.498 & 29.2 & 0.2566 \\
$1,000,000$ & 500 & 0.050 & 31.6 & 0.3536 \\
$1,000,000$ & 50 & 0.005 & 33.9 & 0.0707 \\
$1,000,000$ & 0 & 0 & NA & NA \\
0 & 50,000 & 100 & 24.2 & 0.2887 \\
\hline
\end{tabular}

Real-time PCR results for single-cell suspension mixtures used to create a standardized curve. ASC, adipose-derived stromal/stem cell; CT, cycle threshold; NA, not available; STD, = Standard Deviation.

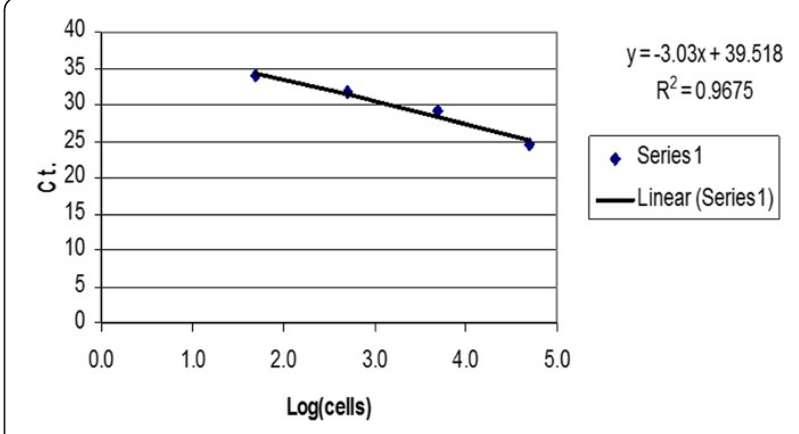

Figure 1 Standard sample of cells mixture analyzed using real-time PCR. Ct, cycle threshold.

\section{Histology and immunohistochemistry}

Tissue sections were stained with hematoxylin and eosin, and representative sections were prepared for immunohistochemical staining by deparaffinization with xylene and rehydration through a graded ethanol series. A heat-mediated antigen retrieval technique that included a 20-minute boil in $0.01 \mathrm{M}$ citrate buffer, $\mathrm{pH} 6.0$ (Fisher Scientific, Waltham, MA, USA) was used. After cooling for 1 hour, two separate 5-minute washes in Tris-buffered saline/Tween20 pH 7.4 (Trizma base; Sigma; Tween20; J. T. Baker, St Louis, MO, USA), followed by two 5-minute washes in phosphate-buffered saline were performed. The sections were incubated in $2 \%$ horse serum (Sigma, Austin, TX, USA) prepared in phosphate-buffered saline with $0.5 \%$ gelatin from cold water fish skin, to inhibit nonspecific binding of the primary antibody. Following incubation in blocking serum, the sections were incubated in primary antibody rat anti-mouse Mac-2 (Cedarlane, Burlington, ON, Canada), dilution 1:1,000, in a humidified chamber for 1 hour. Following this, two 5-minute washes in $0.5 \%$ gelatin from cold water fish skin/phosphate-buffered saline were performed. The sections were then incubated in secondary antibody Alexa Fluor 488 donkey anti-rat (Invitrogen, Carlsbad, CA, USA) for 1 hour in a humidified chamber at room temperature. This was followed by three 5-minute washes in phosphate-buffered saline, prior to aqueous mounting (Fisher Scientific).

The immunostained slides were imaged using a confocal Nikon Eclipse TE 2000-E2 microscope (Nikon, Melville, NY, USA) equipped with a $60 \times$ Nikon oil immersion objective

\section{Statistical analysis}

The percentage of ERV-3 was obtained with real-time PCR as the fraction of ERV-3 remaining at the implantation site on days 3, 10 and 21. Data were analyzed using SPSS 19.0 (IBM Corp. Released 2010. IBM SPSS Statistics for Windows, Version 19.0. Armonk, NY, USA). Using an independent-samples $t$ test, we compared the 
Table 2 Survival of adipose-derived stromal/stem cells

\begin{tabular}{|c|c|c|c|c|c|c|c|c|}
\hline \multicolumn{5}{|c|}{ Immunocompetent mice } & \multicolumn{4}{|c|}{ Immunocompromised mice } \\
\hline \multirow[t]{2}{*}{ \% ERV-3 } & \multicolumn{2}{|c|}{ Viable injection } & \multicolumn{2}{|c|}{ Nonviable injection } & \multicolumn{2}{|c|}{ Viable injection } & \multicolumn{2}{|c|}{ Nonviable injection } \\
\hline & Spheroids & Cells & Spheroids & Cells & Spheroids & Cells & Spheroids & Cells \\
\hline Day $3(n=3)$ & $57.5 \pm 3.9$ & $55.6 \pm 14.7$ & $0.9 \pm 0.8$ & 0 & $90.6 \pm 8.2$ & $87.6 \pm 6.8$ & $0.1 \pm 0.03$ & $0.9 \pm 0.3$ \\
\hline Day $10(n=3)$ & $7.1 \pm 3.0$ & 0 & 0 & 0 & $11.7 \pm 0.8$ & $12.1 \pm 1.3$ & 0 & 0 \\
\hline Day $21(n=3)$ & 0 & 0 & 0 & 0 & $4.8 \pm 0.6$ & $6.3 \pm 0.9$ & 0 & 0 \\
\hline
\end{tabular}

Percent survival of adipose-derived stromal/stem cells (\% ERV-3) measured with real-time PCR. Values represent mean \pm standard error of $n=3$. ERV-3, endogenous retrovirus 3.

percent ERV-3 between the ASCs injected as a cell suspension versus spheroids for different time points (days 3,10 and 21), separately for the two murine species. We also compared the percent ERV-3 between immunocompetent mice versus immunocompromised mice for the time points studied.

\section{Results}

Animal survival

All mice survived the surgical procedure of cell implantation. Out of 72 treated animals, 71 survived to experimental endpoint. One athymic NCr-nu/nu mouse injected with spheroids died on day 7 .

\section{PCR for adipose-derived stromal/stem cell presence and} quantification

Real-time PCR was performed using ERV-3 amplification to assess human cell presence in harvested tissue specimens from injection/implantation sites. The persistence of human ASCs is correlated to the percent ERV-3 amplification [13-15], and our study shows that when viable ASCs are injected in vivo, an increased percentage of cells can be detected relative to nonviable ASC implants, regardless of formulation (cell suspension versus spheroids). As expected, the number of detectable ASCs implanted as either cell suspension or spheroids was greater in immunocompromised mice at all time points as compared with immunocompetent mice, with statistically significant differences on days 10 and 21 after implantation $(P<0.05)$. However, there were no statistical differences in the PCR detection of ASCs over time when implanted as cell suspension compared with those implanted as three-dimensional spheroids (Table 2, Figure 2).

\section{Histology and immunohistochemistry}

On gross observation, the DiI-labeled implants were readily identifiable as pink blushes at the implantation

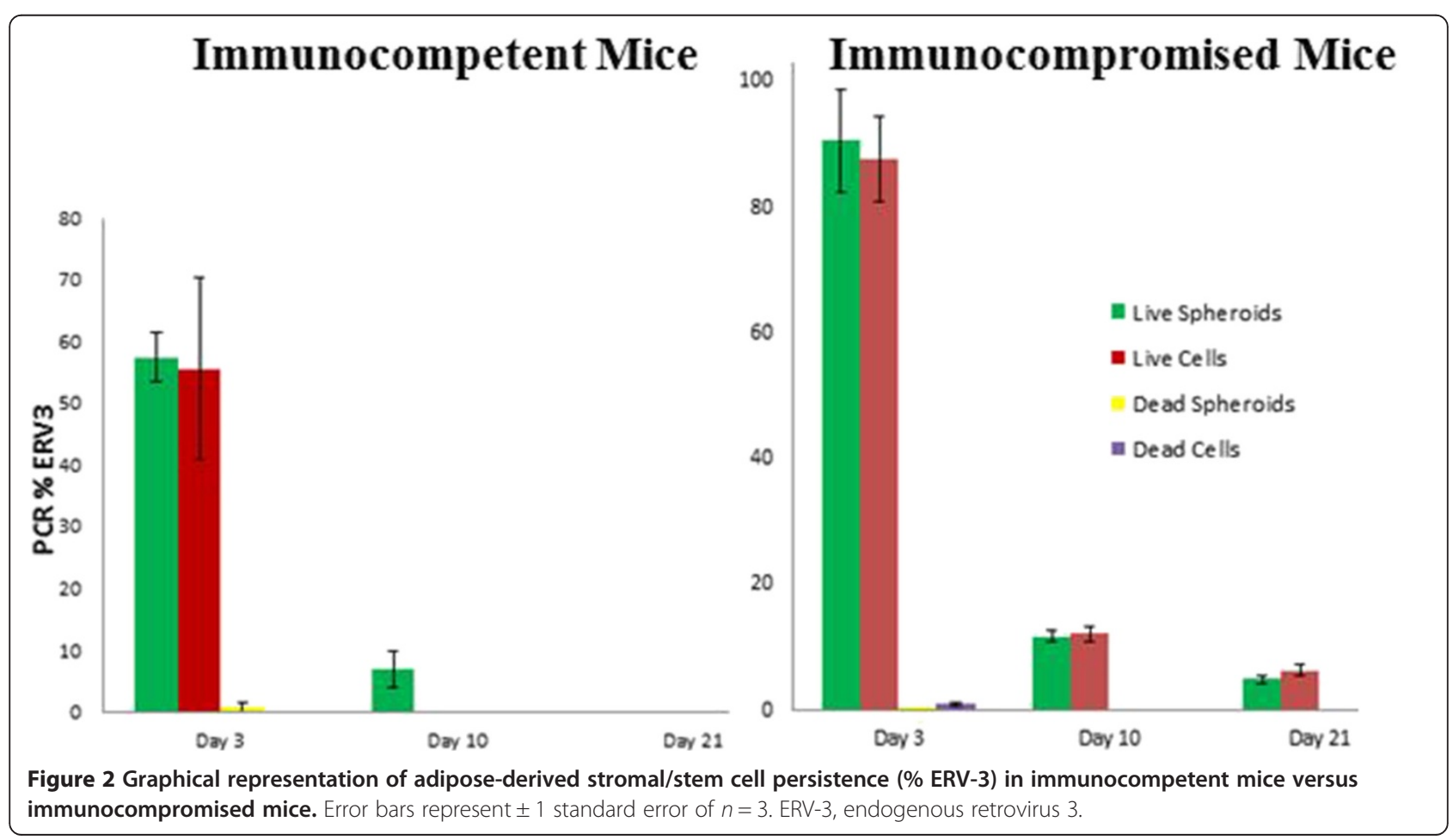


site throughout the entire study period, although the intensity subjectively waned with later time points. Using fluorescent microscopy, the DiI-labeled human adiposederived cell implants were readily visible as a fluorescent signal that was localized to the region of implantation for the entire 3-week study period. The fluorescent signal was easily detectable with a high signal-to-noise ratio. The fluorescent signal arising from nonviable cell implants was indistinguishable from that arising from viable cell implants (Figures 3 and 4). Detection of human cells using highly specific PCR-mediated methods showed that $<1 \%$ of implanted nonviable cells were detectable at the implantation site on day 3 despite the presence of a robust fluorescent signal for up to 3 weeks. On the other hand, up to $90 \%$ of implanted viable cells were detectable by PCR on day 3 , but this rapidly declined to $<5 \%$ by day 21 , once again despite the presence of a robust fluorescent signal. In all cases, the implanted cells were noted to generate a robust macrophage infiltrate, seen with Mac-2 immunostaining that co-localized with the DiI fluorescent signal (Figures 5 and 6).

\section{Discussion}

A variety of cell-based therapies stand at the threshold of changing the way medicine is practiced. A growing body of evidence now suggests that cell therapies act primarily via paracrine effects [16,17]. However, questions remain unanswered as to how many cells are needed and how long such cells are needed at a given site in order to effect a reproducible response. To answer these questions, reliable quantitative methods are required that are both specific and sensitive. In other words, one of several important issues related to the future understanding and translation of such therapies to the clinic pertains to quantifying and optimizing cell survival and biodistribution after cell delivery. This challenge remains critical even for

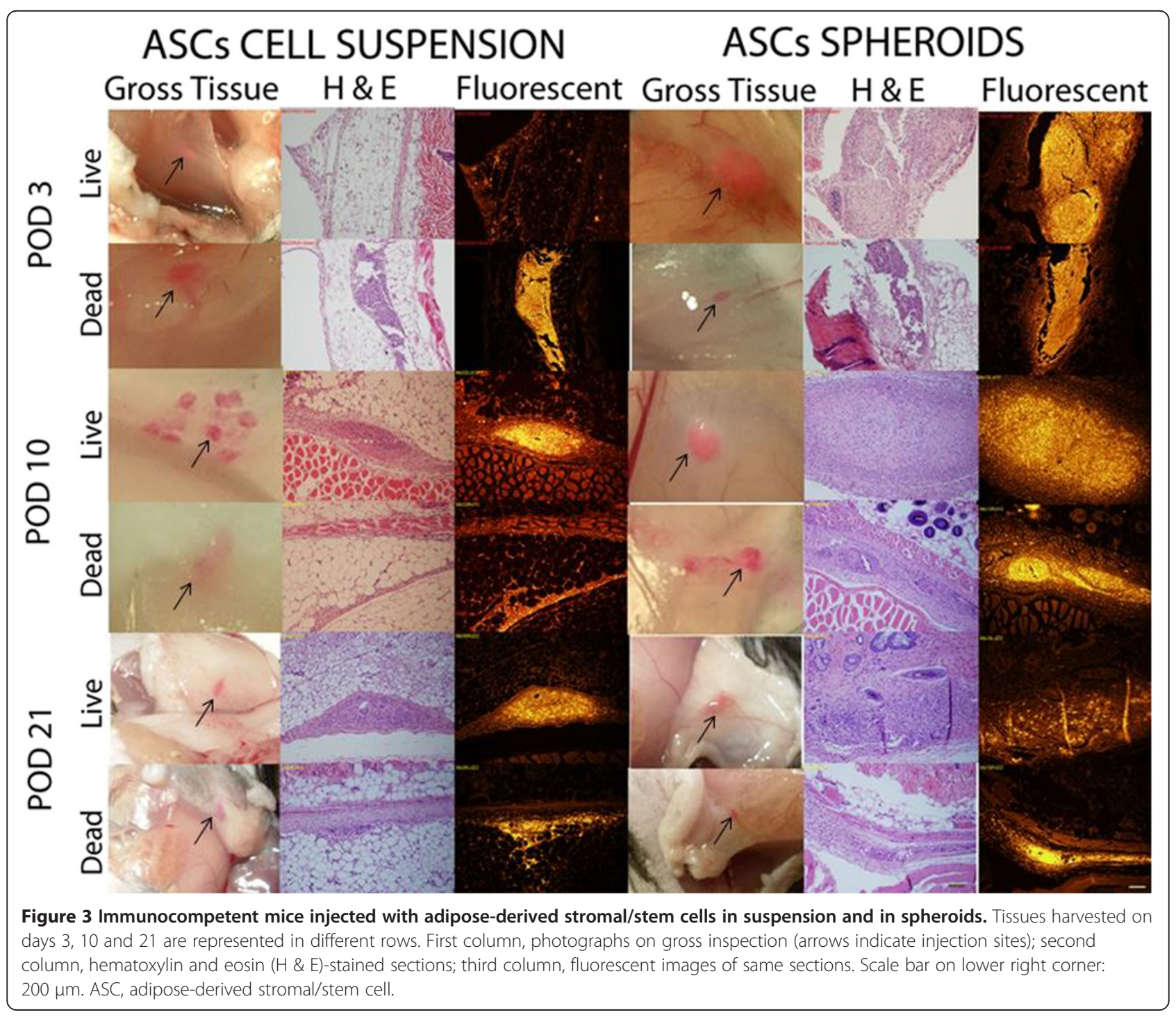




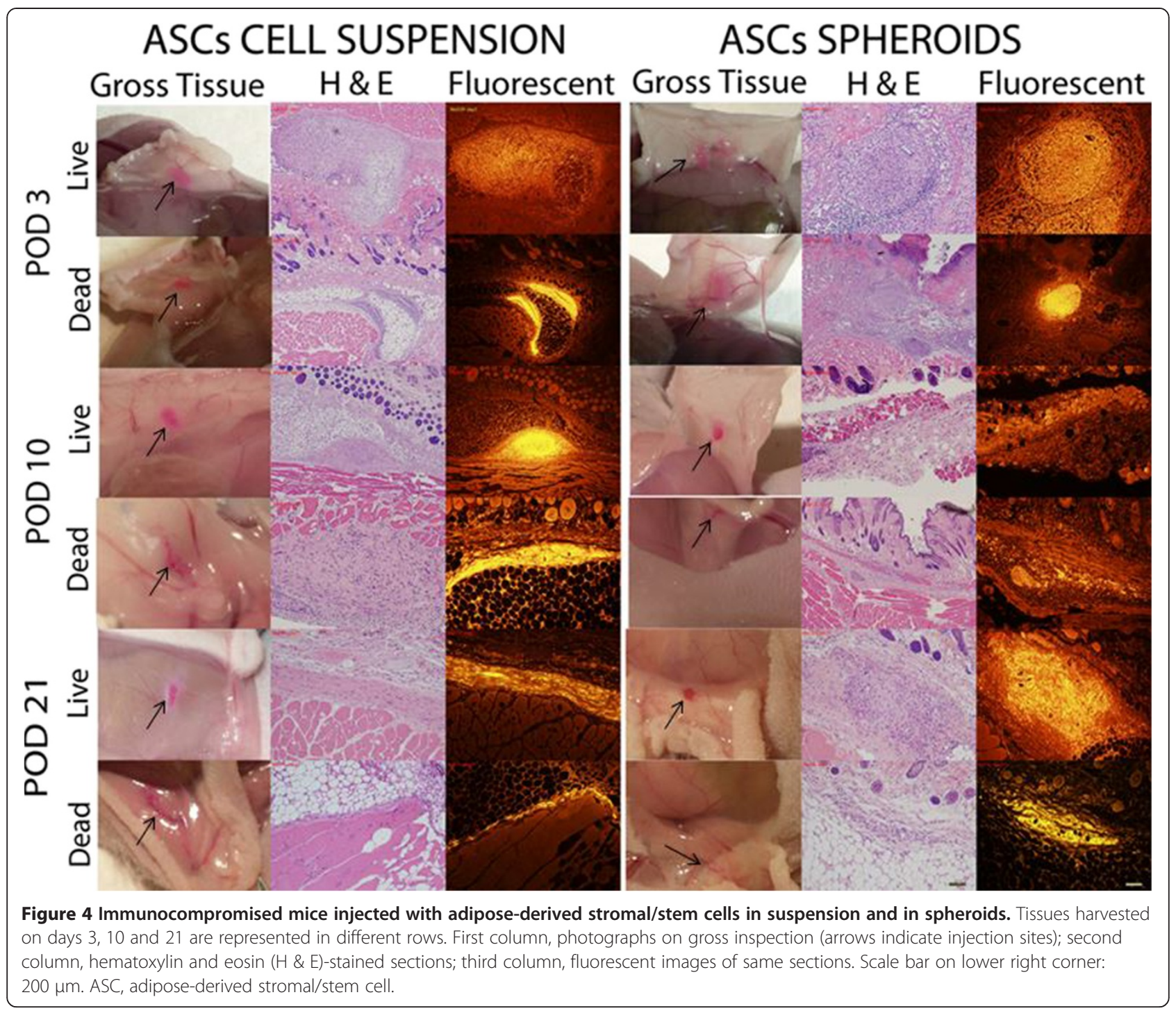

pivotal preclinical studies that are necessary to support the safety and efficacy of emerging therapies.

Given the growing interest in exploiting adipose tissue as an abundant cell source, our objective was to evaluate human ASC persistence after implantation into immunocompetent and immunocompromised mice using fluorescent membrane labeling techniques for histological tracking, and PCR detection of human-specific genetic sequences for confirmatory and quantitative analysis. Both sensitive and specific, real-time PCR for ERV-3 detects as few as 50 human cells in 1,000,000 murine cells $(0.005 \%)$ $[14,15]$, compared with detection of 5,000 cells/organ reported in green fluorescent protein studies [18]. ERV-3 is present in the human genome as only one copy per cell, on chromosome $7[13,14]$. As such, ERV-3 can be used to detect and quantify the presence of human cells, and do so without need for cellular manipulation. However, it does not provide histological or morphological data. Our data show that $<1 \%$ of nonviable ASCs implanted were detectable by PCR detection of ERV-3, 72 hours after implantation. Although detection of ERV-3 does not give a direct confirmation of cell viability, it is conceivable that a close correlation does exist between cell viability and the ability to detect a specific DNA sequence with PCR. It is known, for example, that large DNA fragmentation occurs as early as 5 minutes after the onset of apoptosis. Further breakdown into smaller fragments continues over 2 to 24 hours $[19,20]$.

Based on our PCR data, it appears that human ASCs are cleared quite rapidly in the murine strains used in this study, with clearance in immunocompetent mice occurring faster than in immunocompromised mice. More specifically, nearly $90 \%$ of cells that were viable at injection are undetectable by day 10 , and only $5 \%$ are detectable at the implant site at 21 days. It is possible that a majority of the cells migrate away from the injection site 


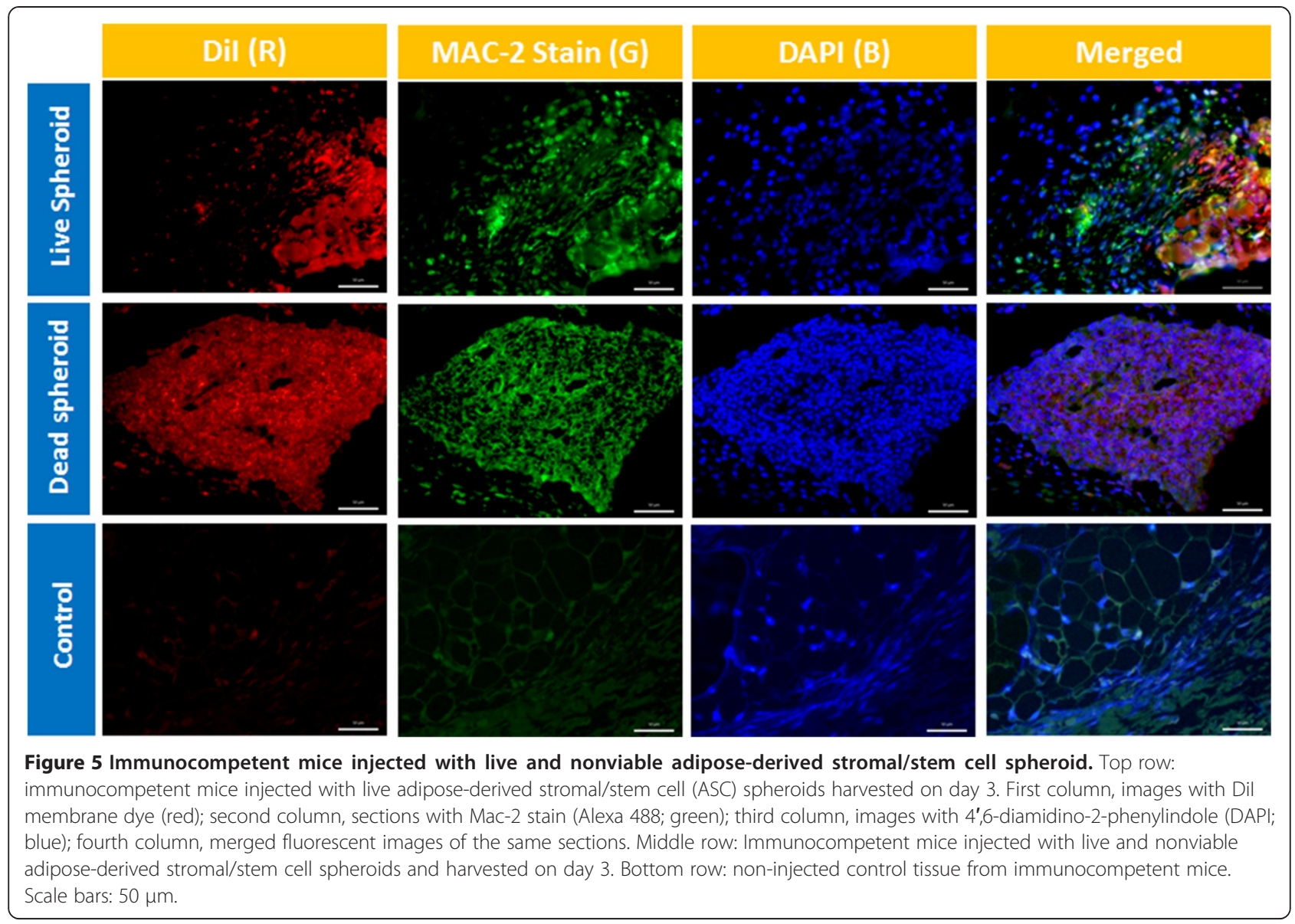

by this early time point. However, the robust fluorescent (DiI) signal that is visible at the implantation sites (Figures 3, 4 and 7) argues strongly against this possibility.

Somewhat surprisingly, human cell clearance also appears to be rapid in the immunocompromised Athymic $\mathrm{NCr}-\mathrm{nu}$ /nu mouse model, raising concern for suitability of this strain for xenogeneic studies. The rapid undetectability of human cells in vivo is associated with a robust infiltration of host macrophages - even in the athymic mouse. Although mature $\mathrm{T}$ cells are missing in athymic mice, the B cells, dendritic cells and granulocytes are all relatively intact, and there is a compensatory increase in macrophages and natural killer cell activity [21]. The colocalization of Mac-2 staining with fluorescent DiI label/ signal suggests strongly that ASC implantation stimulates a rapid and robust macrophage infiltrate which correlates with decreasing (human) cell numbers as measured by ERV-3 quantification (Table 2). Indeed, our results show that a significant amount of Dil signal is located within Mac-2 stained macrophages (Figures 5 and 6). The DiI fluorescent signal remained visible throughout the entirety of our study time period; however, it appears that human ASC-related dye transfer and/or persistence can occur in the context of macrophage-mediated phagocytosis of human cells [22,23], via the exchange of membrane microdomains [3], and/or via microvesicle and/or exosome $[2,24]$ transfer. It is possible that the fluorescent DiI stain is itself immunogenic and may accelerate the engulfment of human cells. There could also be tissuespecific and/or species-specific differences in the macrophage response to implanted cells. In this study, we injected human ASCs subcutaneously and into the inguinal fat pad. Adipose tissue is known to contain resident macrophages and can also efficiently recruit additional macrophages when inflammatory stimuli arise [25-27]. It is conceivable that in other tissues the time course or extent of this phenomenon may look different. In addition to tissue-specific responses, it is unclear whether our findings would be similar in other immunocompromised murine models, such as the nonobese diabetic/severe combined immunodeficiency mouse model. Both of these variables deserve further interrogation.

This study also compared the persistence of human ASCs delivered as single-cell suspensions versus threedimensional spheroids. Contrary to our original hypothesis, this study did not demonstrate any statistical difference in the persistence of ASCs formulated and 

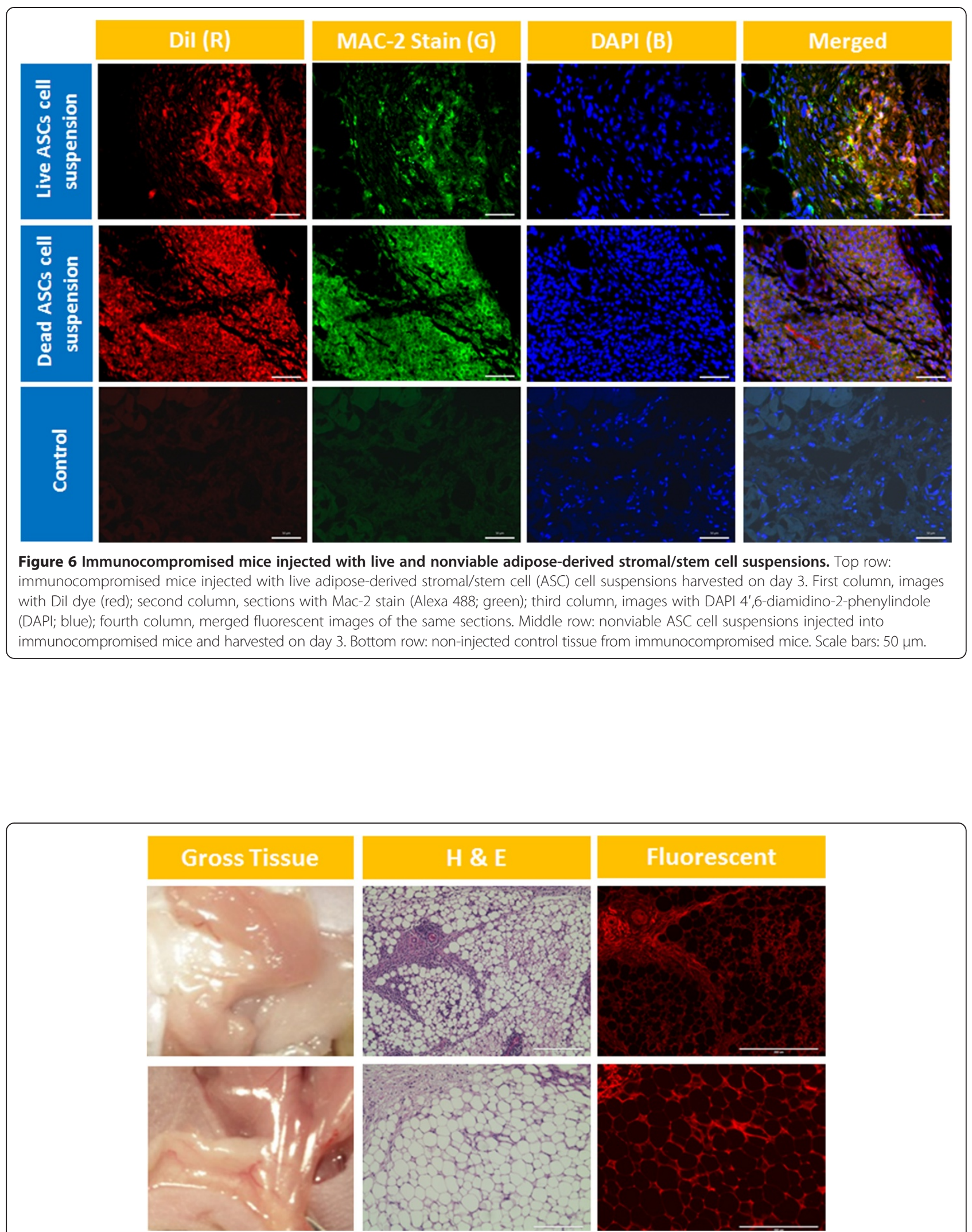

Figure 7 Non-injected control tissues seen on gross inspection, hematoxylin and eosin stain and fluorescent microscopy. Top row: immunocompetent mice. Bottom row: immunocompromised mice. $\mathrm{H} \& \mathrm{E}$, hematoxylin and eosin. 
Table 3 Previous studies of implanted cell persistence and migration in vivo

\section{Reference Species of donor and recipient}

[4] Donor, human ASCs; Recipient, SCID mice

[3]

Donor, human HL60 cells; recipient, NOD/SCID mice

\section{Method and site of delivery}

$5 \times 10^{6}$ cells injected intramyocardially

in the peri-infarct region

Intravenous injection of $20 \times 10^{6}$ cells stained with DIR. Rest of the cell labeling techniques were studied in vitro

Subcutaneous implantation of cells seeded in collagen scaffold

Donor, MCF7 human breast cancer cells, human cord blood-derived cells, human NeoHep cells, human hepatopancreatic precursors; recipient, NOD/SCID mice

Injection into left lobe of liver, $7.5 \times 10^{5}$ human $5 \times 10^{6}$ MCF7 cells; intrapancreatic injection,

$5 \times 10^{5}$ hepatopancreatic precursor cells;

intracardiac transplantation, $5 \times 10^{5}$

hepatopancreatic precursor cells

[6] Donor, human ASCs; recipient, BALB/C $5 \times 10^{6}$ cells injected i.m. or i.v. nu/nu mice

[28] Donor, sheep MSCs; recipient,

Merino-cross sheep

[32] Donor, human ASCs; recipient, BALB/C nu/nu mice
Intramuscular injection

$10^{6}$ cells injected i.m., s.c., i.v., i.p. or enclosed in a fibrin matrix

\section{Cell labeling method}

Transduction with luciferase, GFP

\section{Findings}

10 weeks: $10 \%$ of the human ASCs were localized at the site of injection for 10 weeks. No migration detected. 3.5\% differentiated into cardiomyocytes or endothelial cells.

Lipophilic dyes: Dil, DiD, DiR, PKH26 2 weeks: lipophilic dyes lead to rapid contamination of neighboring cells. CFSE showed good biocompatibility and staining efficiency and showed little contamination. DDAO was toxic to cells. Quantum dots provided

Amine reactive dye: CFSE, DDAO-SE $\begin{aligned} & \text { DDAO was toxic to cells. Quantum dots provided } \\ & \text { heterogeneous staining that is not suitable for intravital }\end{aligned}$ Nano crystals: quantum dots 705 microscopy (IVM). IRDye 800CW had suboptimal excitation Antibodies: IRDye $800 \mathrm{CW}$ by the $633 \mathrm{~nm}$ lasers used for IVM in this study

BrdU labeling

Dil and red fluorescent

nanoparticles Qdot655

4 weeks: BrdU-labeled ASCs were present but no quantification was done

3 weeks: FISH for human-specific Alu sequence and mouse major satellite showed that though many of Dil-labeled cells were human in origin, some were phagocytosed by murine cells. Qdot655 faded during the FISH procedure.

Transduction with luciferase

Dil labeling and CFSE

Lipofection and electroporation with luciferase, GFP
$75 \%$ of cells were lost in first week, the remainder were stable for up to 32 weeks

Dil-labeled MSCs showed dye retention for 6 weeks. CFSE showed rapid signal loss over 8 days

3 weeks: cells migrated and accumulated at the ventral side. A higher fibrinogen concentration limited cell mobility in the fibrin matrix

ASC, adipose-derived stromal/stem cell; CFSE, carboxyfluorescein succinimidyl ester; FISH, fluorescent in situ hybridization; GFP, green fluorescent protein; i.m., intramuscularly; i.p., intraperitoneally; i.v., intravenously; MSC, mesenchymal stem cell; NOD/SCID, nonobese diabetic/severe combined immunodeficiency; s.c., subcutaneously. 
Table 4 Comparison of contemporary cell tracking methods

\section{Tracking method - mechanism}

GFP, luciferase - DNA transfer can be mediated virally (transduction), via liposomes (lipofection), or by electrical parameters (electroporation/ transfection)

\section{Disadvantages}

- Genetic manipulation of cells may alter their function

- Since they are cytosolic in location; the vector (mRNA/DNA) and/or the protein (GFP) could be transmitted to host cells via fusion elements and/ or microvesicle secretion, resulting in contamination. Hence, there is concern for false positivity and their detection may not equate to viability of donor cells

- Technique requires serial passages, not suitable for use with fresh uncultured cells

- Many mammalian tissues have endogenous fluorescence

$\mathrm{BrdU}$ - this nuclear marker is a thymidine analog that replaces $(3 \mathrm{H})$ thymidine and can penetrate cell membranes to incorporate into newly synthesized DNA strands of actively proliferating cells

Lipophilic dyes (Dil, DiR) -long-chain carbocyanine dyes with long aliphatic tails that incorporate into the lipid regions of the cell membranes

- Optimal labeling requires longer incubation time

- Not suitable for non-invasive methods of detection

- Does not indicate viability

- Cells lose BrdU rapidly with serial passages

- Rapidly contaminates neighboring cells by macrophage-mediated phagocytosis, exchange of membrane microdomains, microvesicle and/or exosome transfer
- May be cytotoxic to cells

- The dye fades with serial passages

Amine reactive probes (CFSE) - these diffuse into cells and react with cytosolic amine-containing residues to form dye-protein adducts that are retained

Nanoparticles - small crystals made up of inorganic molecules; for example, iron oxide, cadmium

Real-time PCR for endogenous retroviral sequence (ERV-3) - the gene is present as a single copy in the human genome and so can be used to detect the presence of transplanted human cells in animal models

FISH detection of human-specific cell surface markers or Alu sequences
- The dye is toxic to ASCs

- Not suitable for in vivo non-invasive imaging

- Does not correlate with viability

- Contamination of neighboring cells can occur via macrophage-mediated phagocytosis, microvesicle and/or exosome transfer

- Can be toxic to cells in high concentration and detection is difficult with low concentrations

- Contamination of neighboring cells can occur via phagocytosis, microvesicles and/or exosome transfer

- Does not correlate with viability

- Not suitable for non-invasive methods of detection

- False positives can occur by macrophage-mediated phagocytosis but this is very low

\footnotetext{
- Alu sequences occur in large numbers in the primate genome, which makes higher likelihood of a false positive detection by transmission to host cells/macrophages via microvesicles/exosomes

- Not suitable for non-invasive methods of detection

- Does not correlate with viability
}

\section{Advantages}

- Human ASCs with GFP or luciferase resume proliferation normally

- Detection is sensitive to in vivo noninvasive bioluminescence imaging

- BrdU labeling has no effects on the ASC differentiation/proliferation and is not cytotoxic

- Easy technique for labeling and identification of cells

- Good staining efficiency

- Photostable, remain resistant for long periods of time

- Can be used for in vivo non-invasive imaging

- Gives a quantitative estimate of number of cells

- Very sensitive and specific

- The gene is already present in the human cells, so there is no need to stain the cells

- The gene is already present in the human cells, so there is no need to stain the cells 
anoikis, and remain more localized and robust than similar cells delivered as a single-cell suspension. However, the ERV-3 data do not support this hypothesis, perhaps because the macrophage infiltrate is similar regardless of the implant formulation.

As summarized in Table 3, a wide variety of cell labeling and tracking methods have been reported in the literature. There is notable discrepancy in the findings of these studies and direct comparison is difficult due to differences in cell source/type, recipient species, cell labeling method and concentration, cell dose, and cell delivery method. For example, Lassailly and colleagues report findings for lipophilic dyes (for example, DiI) similar to ours, but find amine reactive dyes to be biocompatible and associated with little contamination [3]. Weir and colleagues, on the other hand, found that amine reactive dyes displayed rapid signal loss over the course of a week [28]. Vilalta and colleagues reported the persistence of $25 \%$ of human ASCs in athymic mice for up to 32 weeks using virally mediated transduction of luciferase [6], and Bai and colleagues reported the persistence of $10 \%$ of human ASCs in SCID mice using a similar labeling method [4]. Although no single method or technique of cell tracking has been proven optimal, each method ought to be reconsidered in light of the emerging literature on microvesicles, and membrane fusion events.

Microvesicles are produced by most cells, including mesenchymal stem cells and ASCs [1], and they can carry and transmit RNA, DNA, cytosolic proteins, and cell membrane proteins. Moreover, it is known that environmental stressors can stimulate the release of microvesicles from cells [29]. As such, cytosolic and membrane-bound proteins such as luciferase and green fluorescent protein can potentially be transmitted to host cells via fusion events and/or microvesicle secretion, resulting in false positive microenvironmental contamination. Similarly, detection of human cell surface labels such as human leukocyte antigen or of human-specific genetic sequences such as Alu can also be a result of microvesicle-mediated false positive contamination of the microenvironment. Although it remains supposition at this point in time, it is statistically plausible that the more prevalent a given protein or genetic sequence is within a cell, the higher the likelihood it will be transferred to a neighboring cell/ microenvironment via a microvesicle or similar mechanism. By this logic, Alu elements - which are the most abundant transposable elements in the human genome as well as cytosolic proteins such as green fluorescent protein and luciferase, are more likely to be transferred to neighboring cells than is the single DNA copy per cell of ERV-3. This assumption certainly requires further studies to confirm or repute, but is worth consideration when interpreting pertinent studies. A comparison of various cell tracking methods, including their relative advantages and disadvantages, is presented in Table 4.

\section{Conclusion}

In summary, these studies demonstrate that when human ASCs are implanted into the subcutaneous and inguinal fat tissue of mice, almost all cells are undetectable within 3 weeks using sensitive and specific molecular techniques. In contrast, a robust fluorescent signal from commonly used membrane dyes is readily detectable by microscopy - even from nonviable cell implants. The colocalization of DiI signal with the MAC-2 stain revealed that DiI is taken up by macrophages by phagocytosis and perhaps other mechanisms of cell transfer. Although clearance of ASCs occurred faster in immunocompetent mice compared with immunocompromised mice, there were no significant differences in the persistence of ASCs delivered as cell suspensions versus those implanted as spheroids. In short, with the emerging evidence of microvesicle formation by ASCs and other modes of host cell contamination, we propose that ERV-3 detection by PCR is a useful method for detecting and quantifying the presence of human cells in xenogeneic models. Further studies are needed to help delineate the advantages and limitations of the various methods available for cell labeling, tracking, identification, and quantification.

\section{Abbreviations}

ASC: adipose-derived stromal/stem cell; $C_{T}$ : threshold cycle; Dil: membrane dye; ERV-3: endogenous retrovirus 3.

\section{Competing interests}

The authors declare that they have no competing interests.

\section{Authors' contributions}

HA carried out animal experiments, immunohistochemistry, PCR, analyzed the data, and drafted the manuscript. HS conceptualized the project and performed animal experiments and PCR. APS conceptualized the project and performed animal experiments and PCR. NY performed animal experiments and PCR and helped in analysis of data. SMP conceptualized the project and revised the manuscript critically for important intellectual content. AJK conceptualized the project, provided guidance and oversight of the experiments, performed the literature review and helped write and critically revised the manuscript. All authors read and approved the final version of the manuscript.

\section{Acknowledgements}

The authors would like to thank Mr John M Sanders for helping with immunohistochemistry.

\section{Funding source}

This project was funded in part by the Armed Forces Institute of Regenerative Medicine (AFIRM-1; to AJK) and in part by the National Institutes of Health/National Institute of Biomedical Imaging and Bioengineering (to AJK).

\section{Author details}

${ }^{1}$ Department of Pediatric Cardiology, Baylor College of Medicine, Texas Children's Hospital, 6621 Fannin Street, Houston, TX 77030, USA. ${ }^{2}$ Department of Surgery, Division of Plastic and Reconstructive Surgery, University of Florida, 1600 SW Archer Road, Gainesville, FL 32610, USA. ${ }^{3}$ George Washington University Hospital, 2121 I St NW, Washington, DC 20052, USA. Department of Biomedical Engineering, University of Virginia, 
415 Lane Road, Room 2041, Charlottesville, VA 22903, USA. ${ }^{5}$ Department of Plastic Surgery, University of Virginia, 1215 Lee Street, Charlottesville, VA 22903, USA.

Received: 20 April 2014 Revised: 8 December 2014

Accepted: 8 December 2014 Published: 18 December 2014

\section{References}

1. Ogawa R, Tanaka C, Sato M, Nagasaki H, Sugimura K, Okumura K, Nakagawa $Y$, Aoki N: Adipocyte-derived microvesicles contain RNA that is transported into macrophages and might be secreted into blood circulation. Biochem Biophys Res Commun 2010, 398:723-729.

2. Quesenberry PJ, Aliotta JM: Cellular phenotype switching and microvesicles. Adv Drug Deliv Rev 2010, 62:1141-1148.

3. Lassailly F, Griessinger E, Bonnet D: 'Microenvironmental contaminations' induced by fluorescent lipophilic dyes used for noninvasive in vitro and in vivo cell tracking. Blood 2010, 115:5347-5354.

4. Bai X, Yan Y, Coleman M, Wu G, Rabinovich B, Seidensticker M, Alt E: Tracking long-term survival of intramyocardially delivered human adipose tissue-derived stem cells using bioluminescence imaging Mol Imaging Biol 2011, 13:633-645.

5. Meyerrose TE, De Ugarte DA, Hofling AA, Herrbrich PE, Cordonnier TD, Shultz LD, Eagon JC, Wirthlin L, Sands MS, Hedrick MA, Nolta JA: In vivo distribution of human adipose-derived mesenchymal stem cells in novel xenotransplantation models. Stem Cells 2007, 25:220-227.

6. Vilalta M, Dégano IR, Bagó J, Gould D, Santos M, García-Arranz M, Ayats R, Fuster C, Chernajovsky Y, García-Olmo D, Rubio N, Blanco J: Biodistribution, long-term survival, and safety of human adipose tissuederived mesenchymal stem cells transplanted in nude mice by high sensitivity non-invasive bioluminescence imaging. Stem Cells Dev 2008, 17:993-1003.

7. Niemeyer P, Vohrer J, Schmal H, Kasten P, Fellenberg J, Suedkamp NP, Mehlhorn AT: Survival of human mesenchymal stromal cells from bone marrow and adipose tissue after xenogenic transplantation in immunocompetent mice. Cytotherapy 2008, 10:784-795.

8. Katz AJ, Tholpady A, Tholpady SS, Shang H, Ogle RC: Cell surface and transcriptional characterization of human adipose-derived adherent stromal (hADAS) cells. Stem Cells 2005, 23:412-423.

9. Zuk PA, Zhu M, Mizuno H, Huang J, Futrell JW, Katz AJ, Benhaim P, Lorenz HP, Hedrick MH: Multilineage cells from human adipose tissue: implications for cell-based therapies. Tissue Eng 2001, 7:211-228.

10. Parker A, Shang H, Khurgel M, Katz A: Low serum and serum-free culture of multipotential human adipose stem cells. Cytotherapy 2007, 9:637-646.

11. Amos PJ, Kapur SK, Stapor PC, Shang H, Bekiranov S, Khurgel M, Rodeheaver GT, Peirce SM, Katz AJ: Human adipose-derived stromal cells accelerate diabetic wound healing: impact of cell formulation and delivery. Tissue Eng Part A 2010, 16:1595-1606.

12. Kapur SK, Wang X, Shang H, Yun S, Li X, Feng G, Khurgel M, Katz AJ: Human adipose stem cells maintain proliferative, synthetic and multipotential properties when suspension cultured as self-assembling spheroids. Biofabrication 2012, 4:025004

13. O'Connell C, O'Brien S, Nash WG, Cohen M: ERV3, a full-length human endogenous provirus: chromosomal localization and evolutionary relationships. Virology 1984, 138:225-235.

14. Lee ST, Chu K, Kim EH, Jung KH, Lee KB, Sinn DI, Kim SU, Kim M, Roh JK: Quantification of human neural stem cell engraftments in rat brains using ERV-3 real-time PCR. J Neurosci Methods 2006, 157:225-229.

15. Yuan CC, Miley W, Waters D: A quantification of human cells using an ERV-3 real time PCR assay. J Virol Methods 2001, 91:109-117.

16. Gnecchi M, Zhang Z, Ni A, Dzau VJ: Paracrine mechanisms in adult stem cell signaling and therapy. Circ Res 2008, 103:1204-1219.

17. Suga H, Glotzbach JP, Sorkin M, Longaker MT, Gurtner GC: Paracrine mechanism of angiogenesis in adipose-derived stem cell transplantation. Ann Plast Surg 2014, 72:234-241.

18. Niyibizi C, Wang S, Mi Z, Robbins PD: The fate of mesenchymal stem cells transplanted into immunocompetent neonatal mice: implications for skeletal gene therapy via stem cells. Mol Ther 2004, 9:955-963.

19. Sun XM, Cohen GM: Mg(2+)-dependent cleavage of DNA into kilobase pair fragments is responsible for the initial degradation of DNA in apoptosis. J Biol Chem 1994, 269:14857-14860
20. Winter DB, Gearhart PJ, Bohr VA: Homogeneous rate of degradation of nuclear DNA during apoptosis. Nucleic Acids Res 1998, 26:4422-4425.

21. Richmond A, Su Y: Mouse xenograft models vs GEM models for human cancer therapeutics. Dis Model Mech 2008, 1:78-82.

22. Pawelczyk E, Arbab AS, Chaudhry A, Balakumaran A, Robey PG, Frank JA: In vitro model of bromodeoxyuridine or iron oxide nanoparticle uptake by activated macrophages from labeled stem cells: implications for cellular therapy. Stem Cells 2008, 26:1366-1375.

23. Pawelczyk E, Jordan EK, Balakumaran A, Chaudhry A, Gormley N, Smith M, Lewis BK, Childs R, Robey PG, Frank JA: In vivo transfer of intracellular labels from locally implanted bone marrow stromal cells to resident tissue macrophages. PLoS One 2009, 4:e6712.

24. Fevrier B, Raposo G: Exosomes: endosomal-derived vesicles shipping extracellular messages. Curr Opin Cell Biol 2004, 16:415-421.

25. Cinti S, Mitchell G, Barbatelli G, Murano I, Ceresi E, Faloia E, Wang S, Fortier M, Greenberg AS, Obin MS: Adipocyte death defines macrophage localization and function in adipose tissue of obese mice and humans. J Lipid Res 2005, 46:2347-2355.

26. Villena JA, Cousin B, Penicaud L, Casteilla L: Adipose tissues display differential phagocytic and microbicidal activities depending on their localization. Int J Obes Relat Metab Disord 2001, 25:1275-1280.

27. Weisberg SP, McCann D, Desai M, Rosenbaum M, Leibel RL, Ferrante AW Jr: Obesity is associated with macrophage accumulation in adipose tissue. J Clin Invest 2003, 112:1796-1808.

28. Weir C, Morel-Kopp MC, Gill A, Tinworth K, Ladd L, Hunyor SN, Ward C: Mesenchymal stem cells: isolation, characterisation and in vivo fluorescent dye tracking. Heart Lung Circ 2008, 17:395-403.

29. Pap E, Pallinger E, Pasztoi M, Falus A: Highlights of a new type of intercellular communication: microvesicle-based information transfer. Inflamm Res 2009, 58:1-8.

30. Lequeux C, Oni G, Mojallal A, Damour O, Brown SA: Adipose derived stem cells: efficiency, toxicity, stability of BrdU labeling and effects on self-renewal and adipose differentiation. Mol Cell Biochem 2011, 351:65-75.

31. Schormann W, Hammersen FJ, Brulport M, Hermes M, Bauer A, Rudolph C, Schug M, Lehmann T, Nussler A, Ungefroren H, Hutchinson J, Fändrich F, Petersen J, Wursthorn K, Burda MR, Brüstle O, Krishnamurthi K, von Mach M, Hengstler JG: Tracking of human cells in mice. Histochem Cell Biol 2008, 130:329-338

32. Wolbank $S$, Peterbauer A, Wassermann E, Hennerbichler $S$, Voglauer $R$, van Griensven M, Duba HC, Gabriel C, Redl H: Labelling of human adiposederived stem cells for non-invasive in vivo cell tracking. Cell Tissue Bank 2007, 8:163-177.

doi:10.1186/scrt532

Cite this article as: Agrawal et al: Human adipose-derived stromal/stem cells demonstrate short-lived persistence after implantation in both an immunocompetent and an immunocompromised murine model. Stem Cell Research \& Therapy 2014 5:142.

\section{Submit your next manuscript to BioMed Central and take full advantage of:}

- Convenient online submission

- Thorough peer review

- No space constraints or color figure charges

- Immediate publication on acceptance

- Inclusion in PubMed, CAS, Scopus and Google Scholar

- Research which is freely available for redistribution 